\title{
Extended applicability of causal inference to compositional data by reciprocal logarithmic ratio transformation
}

7 Daiki Kumakura ${ }^{1 *}$, Ryo Yamaguchi², Shinji Nakaoka ${ }^{2}$

$9 \quad{ }^{1}$ Department of Transdisciplinary Sciences, Hokkaido University

13 E-mail: dkumakura@eis.hokudai.ac.jp

15 Abstract

16 Understanding state transition caused by several intrinsic and extrinsic factors such as

17 environmental change and increased stress of human activities has been a significant

18 focus in ecological studies. Analysis of time-series data is indispensable to identify a

19 causal relationship between the possible factors and community change. Among several

20 time-series analysis, a nonlinear time series analysis method called empirical dynamic

21 modeling (EDM) has been recently applied to infer causality of community change that

22 arose from intraspecies interactions. EDM allows model-free analysis to estimate the

23 degree of action strength of intraspecific and interspecific interactions at the population

24 level. Convergent cross-mapping (CCM) is an empirical dynamic analysis method used

25 to suggest the existence of causality by reconstructing the state space of a dynamical

26 system from a time series of observations without assuming any explicit mathematical

27 equations. Although CCM allows for inferring directional interpretation of causal

28 relationships from multivariate time series data, one of the major challenges is its non- 
29 applicability to compositional data, a common representation of next generation

30 sequencing data such as microbiome. This study aimed to explore a practical approach

31 applied explicitly to compositional data analysis. More specifically, we propose a

32 heuristic but useful transformation that enables CCM to be applied to compositional

33 data. The proposed transformation has demonstrated its applicability to compositional

34 data equivalent to the conventional CCM to untransformed data. Application of the

35 proposed transformation to sequence-based microbial community profiling data

36 provides reasonable implication to the possible causal relationship during state

37 transition.

Author summary

40 Several types of ecological studies such as trophic changes in lakes, marine plankton

41 communities, forest ecosystems, terrestrial ecosystems, and interactions between plants

42 and soils have employed time-series analyses for identifying the factors that might

43 cause state changes. With the rise of next-generation sequencing, our understanding of

44 these ecosystems is expanding to analyze sequence data. A nonlinear time series

45 analysis method, termed empirical dynamic modeling, has been recently applied for

46 analyzing time-series data. Among different empirical dynamic modeling methods,

47 convergent cross-mapping (CCM) is frequently used to infer a causal relationship.

48 Although CCM enables the directional interpretation of causal relationships, it cannot

49 be applied to compositional data analysis. This study proposes a novel type of

50 transformation, Reciprocal Logarithmic Ratio (RLR) transformation, that enables CCM

51 to be applied to compositional data. With RLR-transformation, CCM results for

52 compositional data are comparable to those for absolute data, and it is confirmed that

53 the transformation is applicable to sequence data as well. The RLR-transformation is

54 expected to provide a better understanding of ecological interactions by estimating

55 causal relationships in compositional data.

\section{Introduction}

58 Various types of ecological studies, such as trophic changes in lakes [1], marine

59 plankton communities [2], forest ecosystems [3], terrestrial ecosystems [4], and

60 interactions between plants and soils [5], have performed time-series analyses, with 
61 particular focus on the analysis of identifying state transition using time series data.

62 These studies have mainly focused on determining the factors that may cause state

63 changes. In such studies, network analysis has been extensively used to improve the

64 understanding of interactions among organisms or between organisms and their

65 environments. For example, some studies have focused on the distribution of barnacles

66 in reef intertidal zones [6], predator-prey relationships between fish and plankton [7],

67 the interaction between trophic and non-trophic interactions affecting biological

68 communities [8], and the network structure between plants and bacteria [9]. On the

69 other hand, existing network analysis methods are less applied to infer causal

70 relationships because of the following reasons. First, undirected graphs, as a typical

71 representation of interaction among elements restrict in network analysis, identify the

72 cause and result of the relationship among elements. Second, most interactions are

73 reconstructed from co-occurrence observations, essentially unidirectional without

74 considering any intervention. Third, when analyzing real-time data of complex

75 ecosystems, determining the main inducers of ecological changes and the direction of

76 the interactions between factors is difficult without using temporal network analysis

77 methods [10].

79 Recently, a nonlinear time series analysis method called empirical dynamic modeling

80 (EDM) has been applied for analyzing time series data [11]. EDM has several

81 characteristic features: It determines the complexity (dimensionality) of a system,

82 distinguishing nonlinear dynamical systems from linear stochastic systems, and

83 quantifying nonlinearity (i.e., state dependence), determining causal variables,

84 forecasting, tracking the strength and sign of interaction, and exploring the scenario of

85 external perturbation [12]. EDM also allows model-free analysis of community changes

86 to estimate the degree of action strength of intraspecific and interspecific interactions at

87 the population level. Among different types of EDM methods, convergent cross-

88 mapping $(\mathrm{CCM})$ provides inference for causal relationships by reconstructing the state

89 space of a system from time-series data without assuming any mathematical equations.

90 Specifically, CCM reconstructs an attractor of a dynamical system by applying Takens'

91 embedding theorem to time series data that are assumed to be generated from a

92 nonlinear dynamical system [13]. The causal relationships (i.e., changing one leads to a

93 change in the other) are analogized between the attractors reconstructed from more than

94 two variables of multivariable time series data [14]. Although CCM enables directional

95 interpretation of causal relationships between series, users face several difficulties. For

96 example, its inapplicability to compositional data analysis is a major limitation. 
97 Microbial community profiling based on sequence data uses compositional data. Here

98 we explain why careful consideration is required to infer directionality between a

99 couple of compositional time-series datasets. An intuitive explanation is as follows.

100 This may be because $x_{1}$ and $x_{2}$ were $x_{1}$ and $A-x_{1}$ (A: constant) relationships,

101 called the constant sum constraint, so in the case of the relationship $x_{1} \rightarrow x_{2}$, the

102 constant sum constraint causes the information in $x_{2}$ to contain $x_{1}$, even though the

103 information in $x_{2}$ would not normally be contained in the information in $x_{1}$. As a

104 result, opposite directionality is generated by compositions when it should be

105 unidirectional; hence, there is a possibility of false detection. In this study, we

106 empirically show that CCM is still applied to compositional data is if constant sum

107 constraint is appropriately released.

108

109 In this study, we focus on transformation and scaling, which give rise to a new

110 opportunity to extend the applicability of the CCM method to compositional data. More

111 specifically, we propose a new transformation and scaling method, that we call

112 Reciprocal Logarithmic Ratio (RLR) transformation. The organization of the present

113 paper is as follows. In the next section, as a validation, we show that the CCM with

114 RLR-transformation for compositional data gives comparable results to those of the

115 conventional CCM for absolute data. Furthermore, we demonstrate that the CCM with

116 RLR transformation can be applied to sequence data that are commonly compositional.

117 Based on the validity of our proposed approach, we apply RLR transformation to a

118 microbiome dataset and obtain biological implications for causal relationship of

119 microbial species interactions.

\section{Results}

122 In the case of community structure analysis using 16S rRNA genes, the data output by

123 conventional next-generation sequencers sample only a part of the community, and

124 therefore cannot deal with the absolute amount of species number in the community. In

125 addition, primers are used during sequencing to amplify nucleic acids to enable the

126 detection of DNA fragments, so when comparing the obtained samples, the relative

127 amounts should be compared. Hence, sequence data output should be often analyzed as

128 compositional data, lending to the possibility of detecting false causal relationships and

129 interactions by CCM as intuitively explained above. Considering the possibility of false

130 detection, the conventional CCM can only be applied to count data perse, limiting its 
131 application scope. There exists a reasonable approach to uncover the limitation. For

132 example, sequence data can be weighted by the number of copies of DNA. More

133 specifically, the use of digital PCR allows for absolute quantification [15]. However,

134 this method is in general costed to implement in every sequencing, and it is still difficult

135 to analyze data that are not subjected to this quantification method. Therefore, since the

136 conventional CCM cannot be applied to compositional data, including sequence data,

137 the current scope of CCM application is restricted to estimate causal relationships only

138 for absolute count data.

139

140 In this study, CCM was applied to data that was subject to various transformations. The 141 centered log-ratio (clr) transformation introduced by Aitchison [16] is widely used in

142 various fields [17-25]. The Reciprocal Logarithmic Ratio (RLR) transformation is a

143 novel transformation proposed in this study. Assume that the compositional data consist

144 of two variables, denoted by $X$ and $Y$. This transformation transforms and scales $X$

145 and $Y$ data $(X+Y=1)$ into $-\frac{1}{1+\log \frac{Y}{X}}$ and $-\frac{1}{1+\log \frac{X}{Y}}$, respectively. For these

146 transformed variables, results of CCM were compared based on Cross Map Skill (CMS)

147 and $\omega$. CMS is the correlation coefficient between the predicted value and measured

148 value of the other, while $\omega$ is the difference of CMS. The RLR transformation is tested

149 on variable types of datasets: reference synthetic data and reference real datasets are

150 used for validation, while a sequence dataset is used to demonstrate the references of the

151 RLR transform to infer a causal relationship. A model system of nonlinear dynamics

152 was employed as a source of generating reference synthetic data (see Methods S1 for

153 details). We also examine the effects of changing parameters on reference synthetic data

154 to examine to validate the RLR with reference real datasets; we used the datasets

155 introduced in the CCM proposal paper [14]. Finally, to apply to an insensitivity

156 (robustness) of obtained results a real dataset of sequence dataset, we used the microbial 157 community data [26].

159 Evaluation for the applicability of CCM on the reference synthetic data generated 160 from causality enable model under various transformation.

161 To test whether CCM can be applied to compositional data as well as absolute RLR-

162 transformed absolute data, causal relationships were estimated by CCM on reference

163 synthetic data generated from $x_{1} \rightarrow x_{2}$ model (causality enable model) under several 
164 transformations. Note that a positive value for $\omega$ implies a possible directional

165 causality from $x_{1}$ and $x_{2}$. It was found that CCM can be used for compositional data if

166 we apply RLR-transformation. Fig 1 shows the changes of $\omega$ with respect to the data

167 points. Labels include "Absolute," "Log Absolute," and "Reciprocal Logarithmic Ratio

168 (RLR) Transform." "Absolute" and "Log Absolute" show a decrease of $\omega$ as the time

169 point increased. "Composition," "Log Composition," and "clr Transform" had $\omega$

170 values of zero throughout. Fig 1 (above right-hand corner) shows the average of $\omega$

171 values for each data point. Positive $\omega$ values are found for "Absolute," "Log

172 Absolute," and "RLR-Transform." In contrast, "Composition" and "clr-Transform"

173 values are negative, while "Log Composition" value is slightly positive. For each

174 dataset, the population number changes is shown in Fig S1. "Composition," "Log

175 Composition," and "clr-Transform," and the sum of $x_{1}$ and $x_{2}$, respectively, are

176 always constant at a given point in time, suggesting the existence of a constant sum

177 constraint. By contrast, no constant sum constraint seems to be found for "Absolute,"

178 "Log Absolute," and "RLR-Transform." In conclusion, RLR-transformation can

179 successfully estimate the causal relationship for compositional data as well as for

180 absolute data. 


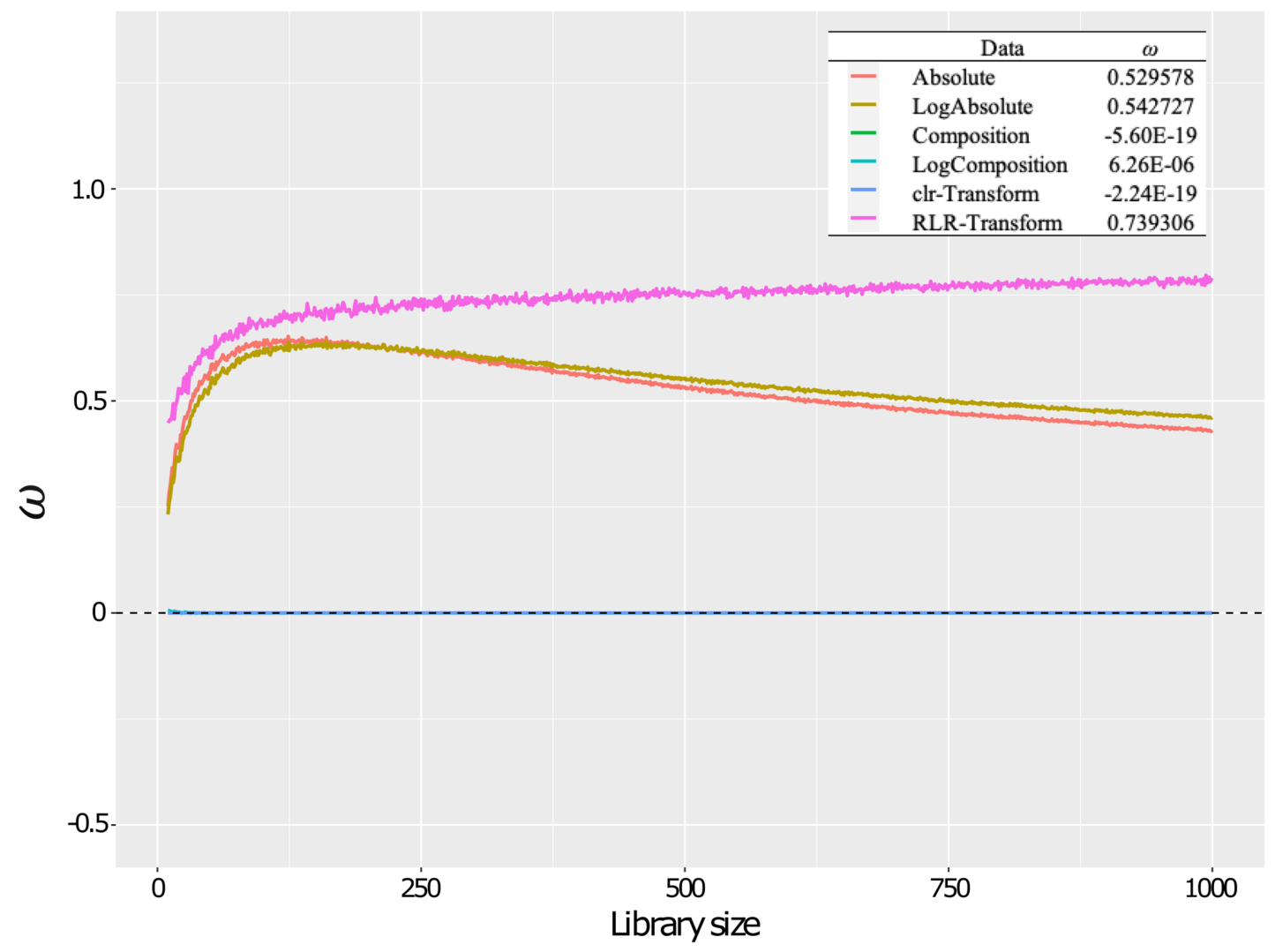

183 Fig 1. RLR-transformation is an equivalent alternative to the conventional CCM

184 for compositional data. Five transformations were performed using "Absolute" as a

185 reference standard, followed by CCM to each transformed dataset. In addition, the

186 average of the correlation coefficients calculated for each transformation is shown in the

187 upper right-hand corner. Indicators of causal relationship shown that "Absolute," "Log

188 Absolute," and "RLR" are positive, while the others are almost zero. The "RLR"

189 exhibits at the same level of "Absolute," even though the data were converted from

190 "Composition."

\section{Dependence of CCM results on parameter}

193 To further investigate whether the RLR transformation can cancel out the inference of constant sum constraint. Parameters were varied to check whether causal relationship can be detected for a wider range of parameter varied, $x_{1} \rightarrow x_{2}$ model was used to compare absolute and compositional data with correlation coefficient $\rho_{x_{2} \widehat{x_{2}}}$. The correlation coefficients between the predicted and measured values of $x_{2}$ from $x_{1}$ are plotted for each parameter in Fig 2. $x_{2}$ is independent of $x_{1}$, no correlation should be 
199 expected between the predicted and measured values of $x_{2}$. However, "Absolute" had

200 positive values for $\rho_{x_{2} \widehat{x_{2}}}$, especially for $r_{2} \geq 3.7 \& 0 \leq \beta_{21} \leq 0.4 \& 3.55 \leq r_{2} \leq$

2013.57 where $\rho_{x_{2} \widehat{x_{2}}}$ was close to 1. For "Composition," $\rho_{x_{2} \widehat{x_{2}}}$ was positive overall. For

202 "RLR-Transform," $\rho_{x_{2} \widehat{x_{2}}}$ was lower than "Absolute." $\rho_{x_{2} \widehat{x_{2}}}$ was close to 1 at $r_{2} \geq 3.8$

$203 \& 0.1 \leq \beta_{21} \leq 0.4 \& 3.55 \leq r_{2} \leq 3.57$. The RLR-transformed data showed the same

204 parameter dependence as the absolute data. Although both Absolute and RLR-

205 transformed data are limitations for parameter dependency [27], RLR-transformed data

206 can be applicable to compositional data constant sum constraint of compositional data.
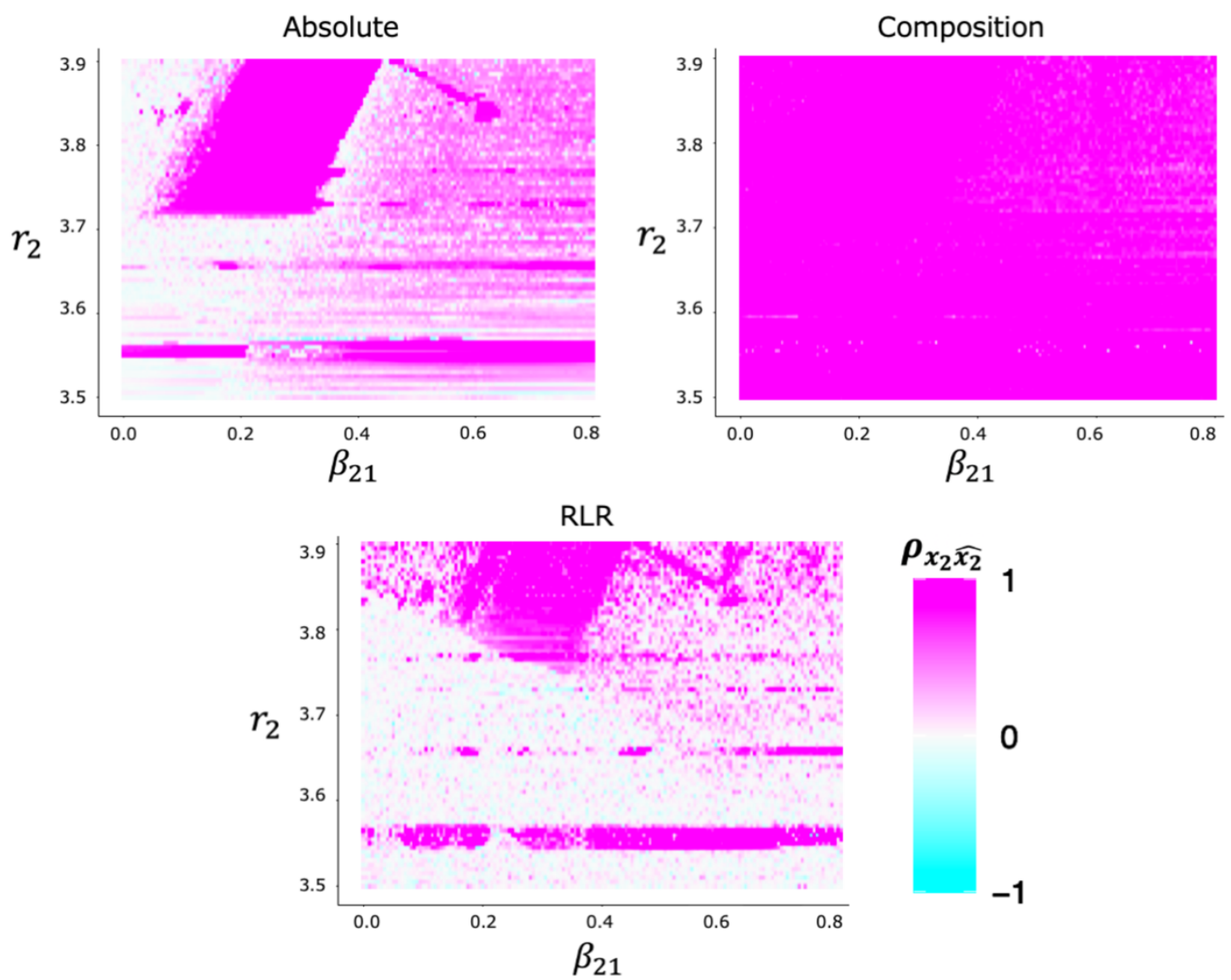

Fig 2. Parameter dependence of each transformation. Each panel represents the

210 correlation coefficients between the observed and predicted values of $x_{2}$ from $x_{1}$ with 211 respect to time points. The results of "Absolute" show a high sensitivity as the CCM 212 results depend on the parameter choice, as explained by Mønster et al. [27]. On the other 213 hand, "Composition" was unchanged (less sensitive) in almost all cases. 


\section{Testing of real data}

216 Since the effectiveness of the RLR-transformation was confirmed on simulation data, in

217 order to validate the applicability of RLR-transformation on reference real data, we used

218 well known real data as another type of reference for the validation of RLR-

219 transformation. The reference real data have been analyzed by CCM and whose

220 causality is well verified. In the analysis, correlation coefficients (correlation

221 coefficients to CMS) and root mean squared error (RMSE) were calculated as the

222 indicator of accuracy between the predicted value and observed value. The validation

223 results show that the RLR-transformed data give similar results to the absolute data. The

224 results of CMS and RMSE comparisons for the three different datasets are shown in Fig

225 3. For the experimental results of the predator-prey system, the results of "Absolute"

226 show that the directionality from Didinium to Paramecium is stronger than that from

227 Paramecium to Didinium (Fig 3A). For RMSE, the value of the Didinium to

228 Paramecium direction is lower than that of the opposite direction. For "Composition,"

229 CMS and RMSE shown the same values in both directions and a constant sum

230 constraint is obtained (Fig 3B). For "RLR Transform," no saturation is observed similar

231 to that observed in "Absolute", but the direction from Didinium to Paramecium is

232 stronger than the opposite direction (Fig 3C). Moreover, the Didinium to Paramecium

233 direction is lower than the opposite direction for RMSE similar to "Absolute." As

234 another example, the relationship between northern anchovy (Engraulis mordax)

235 landings and sea surface temperature (SST) measured at Scripps Pier and Newport Pier

236 in California was also compared in the same way with three datasets (Fig S2). Under the

237 RLR-transformation, similar results to the original data are obtained with CMS and

238 RMSE. In summary, it was confirmed that the RLR-transformation yields the same

239 results as in absolute quantity data, even for real data after compositional

240 transformation. 


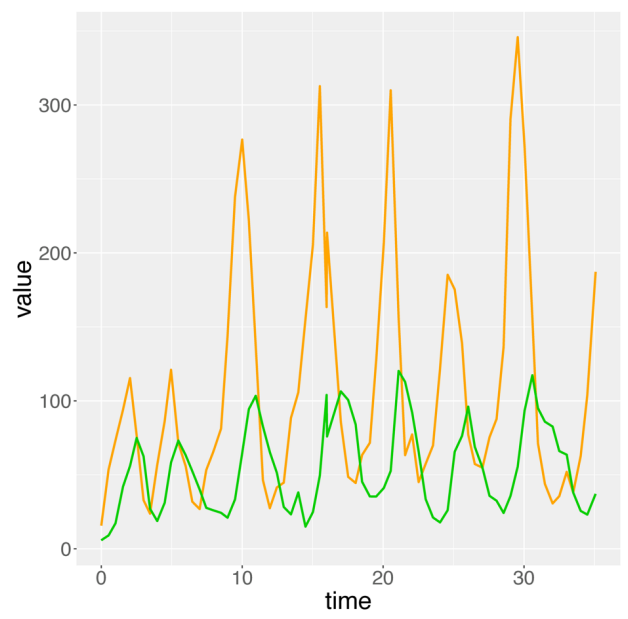

- paramecium

- didinium

- didinium $\rightarrow$ paramecium

- paramecium $\rightarrow$ didinium

\section{Application to real compositional data}
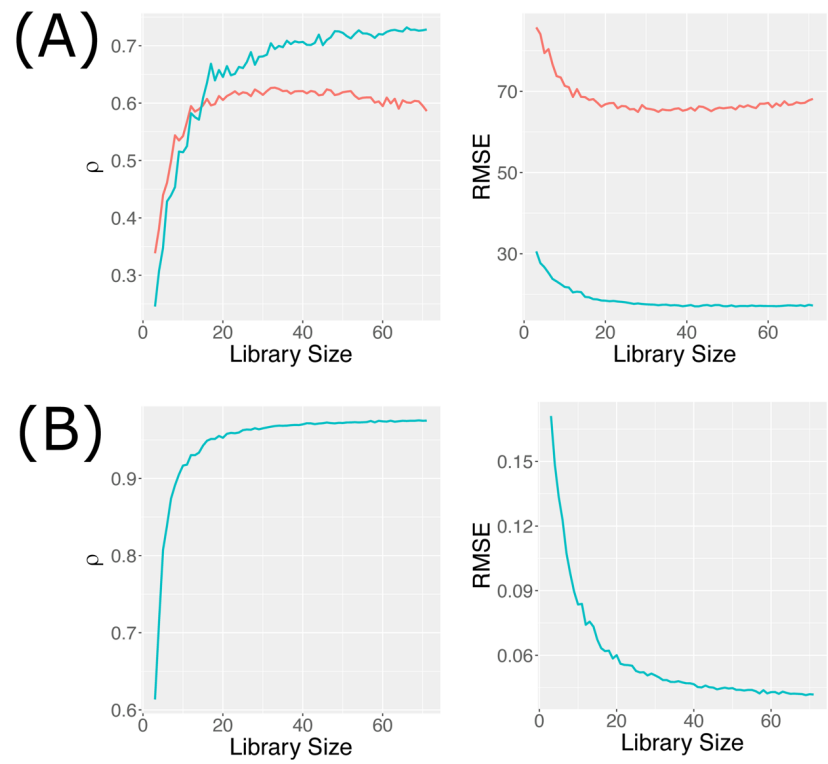

(C)

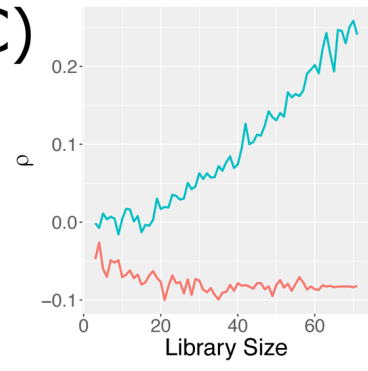

Fig 3. Validation of RLR-transformation on real data. Using "Absolute" as a reference standard, "Composition" shows the same value, and hence, the changes are overlapping. "RLR-Transform" was transformed from the data in "Composition" and showed the same behavior to the results exhibited by "Absolute."

Confirmed the effectiveness of RLR-transformation for real data, we then compared CMS and RMSE between operational taxonomic unit (OTU) raw data represented as compositional, and RLR-transformed data in order to validate the applicability to sequence data. The results shown in figure 4 imply that the RLR-transformed data are consistent with the microbe relationships reported in previous studies, while nontransformed data conflict with previous studies. Of note, the relationship between Clostridium spp. and Ruminococcus gnavus differed before and after the conversion. For Clostridium and Bacteroides spp., the correlation coefficients are similar, but RMSE shows the opposite changes. This implies that there exists a difference in the 
258 CCM results before and after the RLR-transformation. The validation of the RLR-

(A)

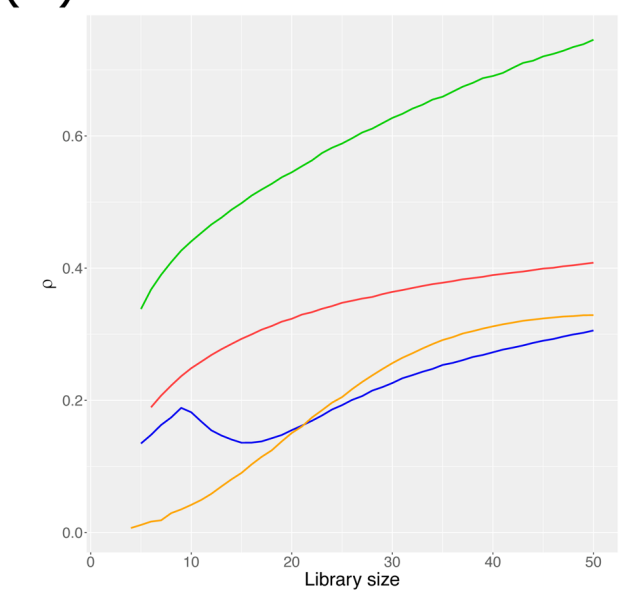

(B)

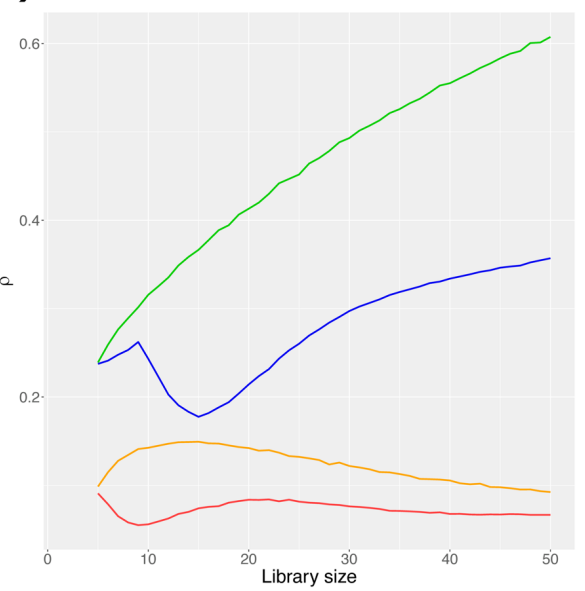

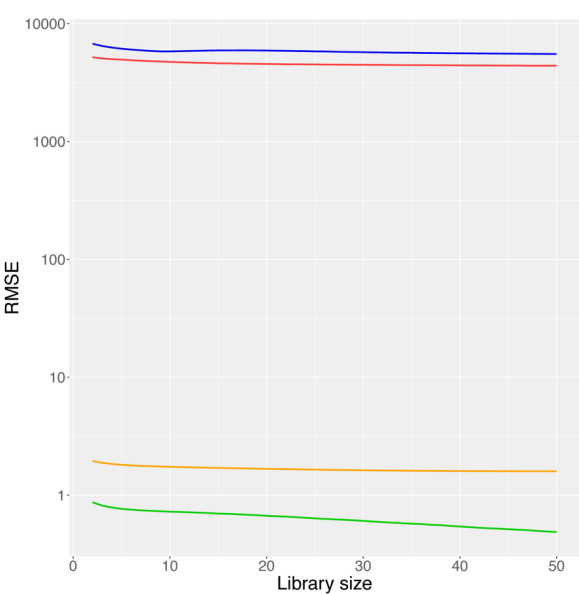

\section{Direction}

- clo $\rightarrow$ rum (seq)

rum $\rightarrow$ clo (seq)

$\underset{\text { rum } \rightarrow \text { clo }}{\text { (RLR }}$ )

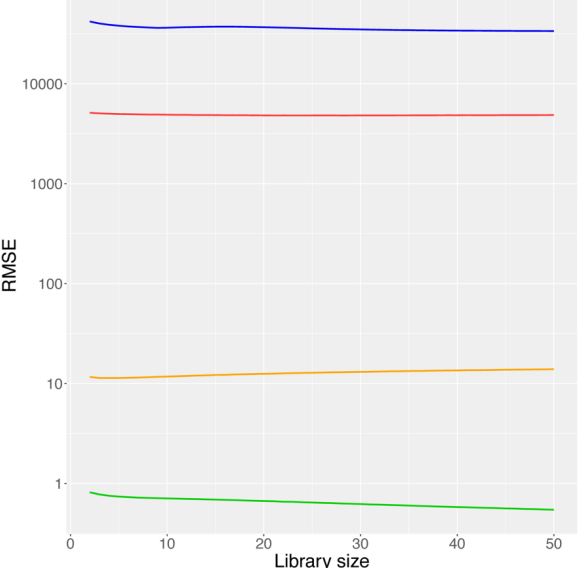

\section{Direction}

- clo $\rightarrow$ bac (seq)

- bac $\rightarrow$ clo (seq)

bac $\rightarrow$ blo (RLR)

262 Fig 4. Application of RLR-transformation to sequence data. Time series datasets of 16S rRNA gene sequencing obtained from fecal samples of an infant are compared with the inferred causal relationship by CCM on the OTU count and after RLR-transformed data [26]. (A) shows the relationship between Clostridium spp. and Ruminococcus gnavus, and (B) shows the relationship between Clostridium and Bacteroides spp. The red and blue lines represent the OTU count data, and the orange and green lines represent the RLR-transformed data used to calculate the CCM. All known relationships are in good agreement with the inferred results by CCM for the RLR-transformed data. 


\section{Discussion}

272 In this study, we examined transformation and scaling can extend the applicability of

273 CCM for compositional data. The proposed method, RLR-transformation has

274 demonstrated its applicability to broader the score of the CCM. In fact, using $x_{1} \rightarrow x_{2}$

275 model, the effectiveness of the RLR-transformation was verified by comparing it with

276 various transformations, and by comparing the dependence on parameters, initial values

277 and variables, and by analyzing the real data. With the RLR-transformation, CCM infers

278 an appropriate causal relationship for compositional data similar to those for the raw

279 data without any transformation. In the following subsections, we discuss the inferred

280 causality of CCM for various data and elements as well as the newly proposed RLR-

281 transformation.

283 Different conversions versus detectability

284 For "Composition" and "clr-Transform," the sum of $x_{1}$ and $x_{2}$ at a given point was

285 always the same, indicating the existence of a constant sum constraint (Fig S1). The

286 constant sum constraint refers to the fact that if the percentage of the D-1 variable is

287 determined when the number of variables in the compositional data is set to $\mathrm{D}$, the

288 percentage of the remaining variables is uniformly determined by subtracting them from

289 the sum of the variables. Compositional data have only one less actual degree of

290 freedom or dimension for the number of variables and is arranged in a D-1 dimensional

291 space [16, 28-29]. For "Absolute" and "RLR Transform" there is no constant sum

292 constraint, and hence, no inter-dependent relationship between $x_{1}$ and $x_{2}$ is not $x_{1}$

293 and $A-x_{1}$ (A: constant) as seen in the result (Fig 1). Therefore, it is suggested that

294 the RLR-transformation released the compositional data from the constant sum

295 constraint and yielded the same CCM results as the absolute data.

\section{Parameters dependence}

298 Since the reference synthetic model does not include $x_{2}$ 's information in $x_{1}$. Therefore, 299 the prediction index of the reverse causality $x_{2} \rightarrow x_{1}$ should be 0 in ideal, and the 
300 correlation coefficient between the predicted value of $x_{2}$ implicated from the data for

$301 x_{1}$ and the observed value must be close to zero. However, depending on the

302 parameters, $\rho_{x_{2} \widehat{x_{2}}}$ as a predictive index changed (Fig 2). In particular, the constant sum

303 constraint inherent to "Composition" resulted in a high correlation even for reverse

304 causality. In fact, Mønster and Sugihara et al. [14, 27] have already reported that CCM

305 does not correctly predict the direction of causality when the coupling is so strong that it

306 results in the synchronization of variables. For the DE-transformed data, it was

307 confirmed that the prediction accuracy was improved to the level of absolute data. This

308 may be due to the fact that the RLR-transformation unlocks the transformed data from

309 the constant sum constraint. In summary, CCM with RLR-transformation is capable of

310 detecting directional causality for compositional data.

312 Verification of the effectiveness of "RLR-Transformation" on reference real data

313 In the real data analysis, the correlation coefficient and RMSE are used as two

314 predictive indices of CCM to test the effectiveness of the RLR-transformation. Fig 3

315 shows that the directionality from Didinium to Paramecium is stronger than the

316 opposite direction in "Absolute," which is similar to the result reported by Sugihara

317 [14]. For "Composition," it is probable that the relationship between Paramecium and

318 Didinium is interdependent because of the constant sum constraint. However, the results

319 for "RLR-Transform" are similar to those of "Absolute," although there is some

320 variation in each library size, and the graphs for RMSE are similar to those of

321 "Absolute." Together with, the results of Fig 3 and Fig S2 suggest that the RLR-

322 transformation can be used to apply CCM to compositional data.

Application of "RLR-Transform" to real data

325 We apply RLR-transformation to microbiome data to infer possible causal relationships

326 among bacterial species in [26]. Fecal samples, collected weekly between 5.5 and 17

327 months old of a female infant who was an asymptomatic carrier of Clostridium spp.,

328 were analyzed by $16 \mathrm{~S}$ rRNA gene sequencing. From the results of Fig 4, after the RLR-

329 transformation of Clostridium spp. and Ruminococcus gnavus, it is speculated that

330 Ruminococcus gnavus is the cause and Clostridium spp. is the result of this relationship.

331 In fact, Ruminococcus gnavus is known to convert lithocholate to ursodeoxycholate and 
332 plays a major role in the formation of ursodeoxycholate in the colon [30].

333 Ursodeoxycholate is known to inhibit spore formation [31]. An increase in

334 Ruminococcus gnavus is known to contribute to the consequent loss of Clostridium

335 spp., which is consistent with the present results [26]. For Clostridium and Bacteroides

336 spp., Bacteroides spp. was the cause, and Clostridium spp. was the result. The gut

337 environment is known to form a barrier to Clostridium spp. colony formation by

338 competing for metabolic substrates and producing inhibitors of Clostridium spp. [32].

339 For example, the relative abundances of Clostridium and Bacteroides spp. are inversely

340 correlated in the human gut [33]. Bacteroides spp. have been shown to form a resistance

341 barrier to Clostridium spp. by competing for monosaccharides such as glucose, N-

342 acetylglucosamine, and succinate and sialic acids [34-38]. Previous studies have shown

343 that an increase in Bacteroides spp. contributes to a decrease in Clostridium spp., which

344 is consistent with the results of the present study.

\section{Summary and perspectives}

347 We demonstrated that an appropriate transform recovers the correct inference of

348 causality even for compositional data. The effectiveness of the RLR-transformation was

349 confirmed through the validation on synthetic and real data. The analysis of sequence

350 data showed that the RLR-transformation can be used to estimate the interactions and

351 causal relationships in a microbial community profiling which is often represented as

352 compositional data. RLR-transformation used in this study is expected to produce

353 results similar to those of absolute count data without causing false positives. The

354 approach presented in this paper can open an opportunity for CCM and other interaction

355 estimation analysis to be widely available for sequence data using NGS.

357 Materials and Methods

358 Model system

359 The logistic map has long been employed as a standard model system of nonlinear

360 dynamics, exhibiting regular periodic behavior as well as deterministic chaos [39]. In

361 particular, generalization of the logistic map allows representing population dynamics

362 of multiple species.

363

$$
x_{i}(t+1)=r_{i} x_{i}(t)\left(1-x_{i}(t)\right)-x_{i}(t) \sum_{j=1}^{n} \beta_{i j} x_{j}(t)
$$


364 Note that interspecific competition is represented by $\beta_{i j} x_{i}(t) x_{j}(t)$, where $\beta_{i j}$ is the strength of competition $(i, j=1,2, \ldots, N)$.

\section{RLR transformation}

368 Reciprocal Logarithmic Ratio (RLR) transformation is defined as a kind of softmax

369 function. Let the compositional data be $X$ and $Y$ with absolute data as $x$ and $y$, and let us consider the transformation of $X$. Now we assume that both $X$ and $Y$ have an effect on each other. The logarithmic odds for the likelihood of $X$ to $Y$ are given by $\log \frac{Y}{X}$. If $X$ is

372 the cause and $Y$ is the result, the log odds represent the likelihood of the result, and if $X$ 373 is affecting $Y$, the odds are high. The log odds are defined as a variant of softmax

374 function, $-\frac{1}{1+\log \left(\frac{Y}{X}\right)}$, and this transformation is called the Reciprocal Logarithmic Ratio 375 (RLR) transformation. This means that $X$ becomes $-\frac{1}{1+\log \left(\frac{Y}{X}\right)}$ by the RLR transformation.

\section{Various transformations}

379 In this study, we generated various transformed datasets to apply CCM and compare 380 results. Transformations include "Absolute" (untransformed), "Composition," "clr381 Transform," and "RLR-Transform." We also examined logarithmic transformations (to 382 cover more different types of transformation, see Methods S1 for details). For

383 "Composition," the values at each time point are obtained as a proportion. The "clr384 Transform" and "RLR-Transform" are applied to "Composition" (Fig 8). For "clr385 Transform," the centered log-ratio transformation (clr) introduced by Aitchison was 386 used [16]. Clr-transformation maps a composition in the $D$-part Aitchison simplex 387 isometrically to a $D-1$ dimensional Euclidean vector. The clr representation of 388 composition $x=\left(x_{1}, \ldots, x_{D}\right)$ is defined as follows:

$$
\operatorname{clr}(x)=\left[\log \left(\frac{x_{1}}{g_{m}(x)}\right), \ldots, \log \left(\frac{x_{D}}{g_{m}(x)}\right)\right],
$$

390 Where $g_{m}(x)=\sqrt[D]{x_{1}, \ldots, x_{D}}$. The sum of the component of $\operatorname{clr}(\mathrm{x})$ is zero [40]. The $\mathrm{clr}$ 391 transforms the composition to the Euclidean sample space and hence provides the 
392 possibility of using standard unconstrained statistical methods for analyzing

393 compositional data [41]. This conversion method was widely used in geology [17-19].

394 In recent years, it has been applied in various fields, such as social sciences [20],

395 archaeology[21], agriculture [22], microbiology [23], ecology [24], and evolution [25].

\section{Convergent cross-mapping}

398 Convergent cross mapping (CCM) is a method for inferring causality in terms of the

399 reconstruction of the state space of a dynamical system from multiple time series data

400 [14]. The basic principle of cross-mapping involves reconstructing system states from

401 two-time series variables and then quantifying the correspondence between them using

402 nearest neighbor forecasting [42]. For two-time series, two attractors are reconstructed

403 by embedding from Takens' theorem. Then, the effect of one on the other is measured

404 by evaluating the error of the correlation coefficient (Cross Map Skill) between the

405 estimated time series and the actual time series. In this study, we checked the

406 nonlinearity of generated time-series data by S-map which is computed by using R

407 package rEDM, selected the embedding dimension at minimum predictive RMSE, and

408 conducted cross-mapping from the two-time series using the "ccm" function in rEDM.

409 The results were visualized as library size on the horizontal axis and Cross Map Skill

410 (CMS) on the vertical axis. In this study, we introduced a new measure of causality, $\omega$,

411 to compare the results of CCM across multiple data sets (Fig 1). Note that $\omega$ represents

412 the CMS difference between the pair of CCM results for each library size. Using this

413 difference, we compared the changes of CMS by CCM in multiple datasets. More

414 specifically, $\omega$ is computed as follows:

415

416

417

418

\section{Validation of CCM using multiple types of data}

420 To validate whether CCM can correctly infer causal relationship, multiple artificial data

421 representing population dynamics of a prey-predator system are generated. First, we

422 considered a two-variable nonlinear deterministic coupled system [14] (see Methods S1

423 for details). The dataset is transformed "Absolute," "Composition", "clr-transform”, and 
424 RLR-transform (see S1 for details). CCM was performed for each type of dataset. Then,

425 the directionality was evaluated by $\omega$, and compered to examine correct detectability.

\section{Verification of parameter dependence}

428 We tested whether the results of CCM were affected by parameter values. The

429 conditions for the various parameters and variables were generated according to

430 Mønster et al. 2017 [27] (see Methods S1for details). The data were calculated

431 according to the conditions, with "Absolute," "Composition," and "RLR-Transform."

432 For verification, the correlation coefficient between the predicted and measured values

433 of $x_{2}$ was used.

\section{Validation of the effectiveness of RLR-transformation on reference data}

436 Using the actual data, we validated "RLR Transform" for CCM applicability compared

437 with "Absolute" and "Composition." For the actual data, we used the two datasets

438 previously employed in [14], and the sequence dataset with fecal microbiota [26]. The

439 first was analyzed using a predator-prey system at the laboratory level, where both the

440 directions of causality exist. This was first studied by Gause in the 1920s and later

441 refined by Veilleux [43], confirming the involvement of Didinium (predator) and

442 Paramecium (prey) [14]. For these data, CCM was applied, and CMS comparison was

443 performed using the optimal embedding dimension. In addition, to evaluate the

444 accuracy of the predictions, the RMSE values were also compared with each dataset.

\section{Application to real data}

447 The microbial community profiling data obtained from fecal samples were used to

448 compare differently. Briefly, 16S rRNA gene sequences are obtained from fifty fecal

449 samples, collected weekly between 5.5 and 17 months old of a female infant who was

450 an asymptomatic carrier of Clostridium spp. Here, we focused our analysis on the

451 relationship between Clostridium spp. and Ruminococcus gnavus, Bifidobacterium

452 bifidum, and Bacteroides spp., which was described in [26]. Microbiome data

453 represented by the operational taxonomy unit (OTU) table were used (see for the detail 
454 [44]). Specifically, the time-series data for Clostridium spp. and Ruminococcus gnavus,

455 Clostridium spp., Bifidobacterium bifidum, and Clostridium spp. and Bacteroides spp.

456 were analyzed and compared with the RLR transformed data, and the results were

457 discussed in addition to what has already been discussed in the previous study [26].

\section{Acknowledgments}

460 This work is supported by JST-Mirai Program Grant Number JPMJMI19B1, the Japan

461 Society for the Promotion of Science (JSPS) Grant-in-Aid (S) $15 \mathrm{H} 0570710$ and (B)

$46218 \mathrm{H} 0266210$, and the Ministry of education, culture sports, science and technology-

463 Japan (MEXT) Ambitious Tenure Track program in life science, Hokkaido University.

\section{References}

466 [1] Lindeman RL. The trophic-dynamic aspect of ecology. Ecology. 1942;23(4): 399-

467417.

468 [2] Margalef R. On certain unifying principles in ecology. Am Nat. 1963;97(897): 357469374.

470 [3] Green DG. Time series and postglacial forest ecology. Quat Res. 1981;15(3): 265-

471277.

472 [4] Odum EP. The strategy of ecosystem development. Science. 1969;164(3877): 262-

473270.

474 [5] Bormann FH, Likens G. Pattern and process in a forested ecosystem: disturbance,

475 development and the steady state based on the Hubbard Brook Ecosystem Study.

476 Springer New York; 1979

477 [6] Connell JH. Effects of competition, predation by Thais Lapillus, and other factors on 478 natural populations of the barnacle Balanus balanoides. Ecol Monogr. 1961;31(1): 61479104.

480 [7] Cohen JE, Tomas J, Stephen RC. Ecological community description using the food 481 web, species abundance, and body size. Proc Natl Acad Sci U S A. 2003;100(4): 17814821786.

483 [8] Kéfi S, Eric LB, Evie AW, Sergio AN, Owen LP, Spencer AW, et al. More than a 484 meal... integrating non-feeding interactions into food webs. Ecol Lett. 2012;15(4): 291485300 . 
486 [9] Toju H, Hirotoshi S, Akifumi ST. Diversity and spatial structure of belowground

487 plant-fungal symbiosis in a mixed subtropical forest of ectomycorrhizal and arbuscular 488 mycorrhizal plants. PloS One. 2014;9(1): e86566.

489 [10] McCann KS, Gellner G. Theoretical ecology: concepts and applications. Oxford:

490 Oxford University Press; 2020.

491 [11] Ushio M, Hsieh Ch, Masuda R, Deyle ER, Ye H, Chang Cw, et al. Fluctuating

492 interaction network and time-varying stability of a natural fish community. Nature.

493 2018;554: 360-363.

494 [12] Chang Cw, Ushio M, Hsieh Ch. Empirical dynamic modeling for beginners. Ecol 495 Res. 2017;32(6): 785-796.

496 [13] Takens F. Detecting strange attractors in turbulence. In Dynamical Systems and

497 Turbulence. Springer Berlin Heidelberg. Warwick; 1980, 366-381.

498 [14] Sugihara G, May R, Ye H, Hsieh Ch, Deyle E, Fogarty M, et al. Detecting 499 causality in complex ecosystems. Science. 2012;338(6106): 496-500.

500 [15] Shiroguchi K, Jia TZ, Sims PA, Xie XS. Digital RNA sequencing minimizes

501 sequence-dependent bias and amplification noise with optimized single-molecule

502 barcodes. Proc Natl Acad Sci U S A. 2012 Jan 24;109(4):1347-52.

503 [16] Aitchison J. The statistical analysis of compositional data. J R Stat Soc Series B

504 Stat Methodol. 1982;44(2): 139-160.

505 [17] Zhou Di, Hanzong C, Youlang L. The logratio approach to the classification of 506 modern sediments and sedimentary environments in Northern South China Sea. Math 507 Geol. 1991;23(2): 157-165.

508 [18] Rollinson HR. Another look at the constant sum problem in geochemistry. Mineral 509 Mag. 1992;56(385): 469-475.

510 [19] Aitchison J. Logratios and natural laws in compositional data analysis. Math Geol. 511 1999;31: 563-580.

512 [20] Katz J, Gary K. A statistical model for multiparty electoral data. Am Polit Sci Rev. $5132020 ; 93$. Available from: https://gking.harvard.edu/files/abs/multiparty-abs.shtml.

514 [21] Heidke JM, Miksa EJ. Correspondence and discriminant analyses of sand and sand 515 temper compositions, tonto basin, Arizona. Archaeometry. 2000;42(2): 273-299.

516 [22] Muldowney D, Connolly J, Keane MG. Compositional data analysis in the study of 517 carcass composition of beef cattle. Livest Prod Sci. 2001;67(3): 241-251.

518 [23] Gloor GB, Macklaim JM, Pawlowsky-Glahn V, and Egozcue JJ. Microbiome 519 datasets are compositional: and this is not optional. Front Microbiol. 2017;8: 2224. 520 [24] Elston DA, Illius AW, Gordon IJ. Assessment of preference among a range of 521 options using log ratio analysis. Ecology. 1996;77(8): 2538-2548. 
522 [25] Brandt M, Heinze J, Schmitt T, Foitzik S. A chemical level in the coevolutionary

523 arms race between an ant social parasite and its hosts. J Evol Biol. 2005; 18: 576-586.

524 [26] Davis MY, Zhang H, Brannan LE, Carman RJ, Boone JH. Rapid change of fecal

525 microbiome and disappearance of Clostridium difficile in a colonized infant after

526 transition from breast milk to cow milk. Microbiome. 2016 Oct 7;4(1):53.

527 [27] Mønster D, Fusaroli R, Tylén K, Roepstorff A, Sherson JF. Causal inference from

528 noisy time-series data - testing the convergent cross-mapping algorithm in the

529 presence of noise and external influence. Future Gener Comput Syst. 2017;73: 52-62.

530 [28] Reyment RA. Compositional data analysis. Terra Nova. 1989;1(1): 29-34.

531 [29] Ohta T, Hiroyoshi A. Problems in compositional data analysis and their solutions. J

532 Geol Soc Japan. 2006;112(3): 173-187.

533 [30] Lee JY, Arai H, Nakamura Y, Fukiya S, Wada M, Yokota A. Contribution of the

$5347 \beta$-hydroxysteroid dehydrogenase from Ruminococcus gnavus N53 to ursodeoxycholic

535 acid formation in the human colon. J Lipid Res. 2013;54(11): 3062-3069.

536 [31] Sorg JA, Sonenshein AL. Chenodeoxycholate is an inhibitor of Clostridium

537 difficile spore germination. J Bacteriol. 2009;191(3): 1115-1117.

538 [32] Britton RA, Young VB. Role of the intestinal microbiota in resistance to

539 colonization by Clostridium difficile. Gastroenterology. 2014;146(6): 1547-1553.

540 [33] Goldberg E, Amir I, Zafran M, Gophna U, Samra Z, Pitlik S, et al. The correlation

541 between Clostridium-difficile infection and human gut concentrations of bacteroidetes

542 phylum and clostridial species. Eur J Clin Microbiol Infect Dis. 2014;33(3): 377-383.

543 [34] Wilson KH, Perini F. Role of competition for nutrients in suppression of

544 Clostridium Difficile by the colonic microflora. Infection and Immunity. 1988;56(10):

$545 \quad 2610-2614$.

546 [35] Reichardt N, Duncan SH, Young P, Belenguer A, Leitch CM, Scott KP, et al.

547 Phylogenetic distribution of three pathways for propionate production within the human

548 gut microbiota. ISME J. 2014;8(6): 1323-1335.

549 [36] Brigham C, Caughlan R, Gallegos R, Dallas MB, Godoy VG, Malamy MH. Sialic 550 acid ( $\mathrm{N}$-acetyl neuraminic acid) utilization by Bacteroides fragilis requires a novel $\mathrm{N}$ -

551 acetyl mannosamine epimerase. J Bacteriol. 2009;191(11): 3629-3638.

552 [37] Hopkins MJ, Macfarlane GT. Changes in predominant bacterial populations in

553 human faeces with age and with Clostridium difficile infection. J Med Microbiol.

554 2002;51(5): 448-454.

555 [38] Hopkins MJ, Macfarlane GT. Nondigestible oligosaccharides enhance bacterial

556 colonization resistance against Clostridium difficile in vitro. Appl Environ Microbiol.

557 2003;69(4): 1920-1927. 
558 [39] May RM. Simple mathematical models with very complicated dynamics. Nature.

559 1976;261(5560): 459-467.

560 [40] Xia Y, Jun S, Ding-Geng C. Statistical analysis of microbiome data with R.

561 Springer, Singapore; 2018

562 [41] Aitchison J, Jim W, Kay J. Possible solution of some essential zero problems in

563 compositional data analysis. 2003. Available from: https://dugi-

564 doc.udg.edu/handle/10256/652.

565 [42] Ye H, Chang Cw, Sugihara G, Kondoh M. Fluctuating interaction network and

566 time-varying stability of a natural fish community. Nature. 2018;554: 360-363.

567 [43] Veilleux BG. An analysis of the predatory interaction between Paramecium and

568 Didinium. J Anim Ecol. 1979;48(3): 787-803.

569 [44] Shinji Nakaoka, Detection of responding factors to external perturbation in a

570 microbial community, Proc. of International Symposium on Nonlinear Theory and Its

571 Applications, NOLTA2017, 116-119.

\section{Supplementary information}

574 Methods S1. Detailed methods for each verification point.

\section{Comparison of logarithmic transformation with several conversions}

576 In this study, we tested comprehensive transformation with several datasets including

577 compositional data. The data output from the equation is transformed to "Absolute" (no

578 transformation), and "Log (Absolute + 1)." For compositional transformed data

579 "Composition," we further transformed to obtain "Log (Composition + 1)," centered

580 log-ratio transform "clr-Transform," and the newly proposed transformation "RLR

581 Transform." Note that "Log (Value + 1)" and "clr-Transform" are major transformations

582 for converting compositional data.

\section{Validation of CCM using multiple types of data}

585 Using the population dynamics model below, we generate 1000 time series datasets of

586 the system with $x_{i}(0)=0.1$ as an initial condition. Parameter values are set as

587 follows:

$$
x_{i}(t+1)=r_{i} x_{i}(t)\left(1-x_{i}(t)\right)-x_{i}(t) \sum_{j=1}^{n} \beta_{i j} x_{j}(t)
$$

$$
i, j=1,2
$$


590

591

592

593

594

595

596

597

598

599

600

601

602

603

604

605

606

607

608

609

610

611

612

613

614

615

61

617

618

619

$$
\begin{array}{ll}
i=1: r_{i}=3.80, & \beta_{i j}=0.02 \\
i=2: r_{i}=3.50, & \beta_{i j}=0.10
\end{array}
$$

\section{Verification of parameter dependence}

For system(1), we set a restriction to equations such that $x_{1}$ consists of $x_{1}$ only, whereas $x_{2}$ consists of $x_{1}$ and $x_{2}$. Synthetic data were generated up to 1400 time points, and 1000 to 1400 time points were used. Parameters and variables are set as follows:

$$
\begin{gathered}
i, j=1,2 \\
i=1: \beta_{i j}=0 \\
r_{1}+r_{2}=7.3984, \quad 3.5 \leq r_{2} \leq 3.9 \\
0 \leq \beta_{21} \leq 0.8 \\
x_{i_{0}}=0.1
\end{gathered}
$$

Appendix S1. Testing of real data on the relationship between anchovy and sea surface temperature.

\section{Material and Methods}

The dataset was analyzed to identify the complex causation in the sardine-anchovy system [14]. The data describe the relationship between northern anchovy (Engraulis mordax) landings and sea surface temperature (SST) measured at Scripps Pier and Newport Pier, California. As the data used in this study included negative values, minmax normalization was performed, and the two datasets were normalized. The conversion formula for min-max normalization is given below:

$$
Z_{S}=\frac{Z-\min (Z)}{\max (Z)-\min (Z)},
$$

where $\mathrm{z}$ is an original value and $Z_{s}$ is the normalized value. The raw data were denoted "Raw," while the compositional data were "Composition," and the data transformed "Composition" to RLR-transformation were denoted as "RLR-Transform," respectively.

617 For these data, CCM was applied, and CMS comparison was performed using the optimal embedding dimension. In addition, to evaluate the accuracy of the predictions, RMSE values were also compared with each dataset. 


\section{Result and Discussion}

622 Considering the analysis results of the complex causation in the sardine-anchovy

623 system, the change of CMS for "Raw" are shown in Fig S2 A. This indicates that sea

624 surface temperature affects the landings of anchovies. For "Composition," CMS and

625 RMSE exhibit the same values in both directions, and a constant sum constraint was

626 obtained (Fig S2 B). For "RLR-Transform," the changes of CMS and RMSE were

627 similar to that of "Raw," and the direction from sea surface temperature to anchovy

628 landings had lower values than that in the opposite direction (Fig S2 C). Fig S2 implies

629 that, for "Composition," the constant sum constraint may be due to the equally strength

630 of 2 variables. For "RLR-Transform," CMS and RMSE showed the same changes as

631 obtained for "Raw."
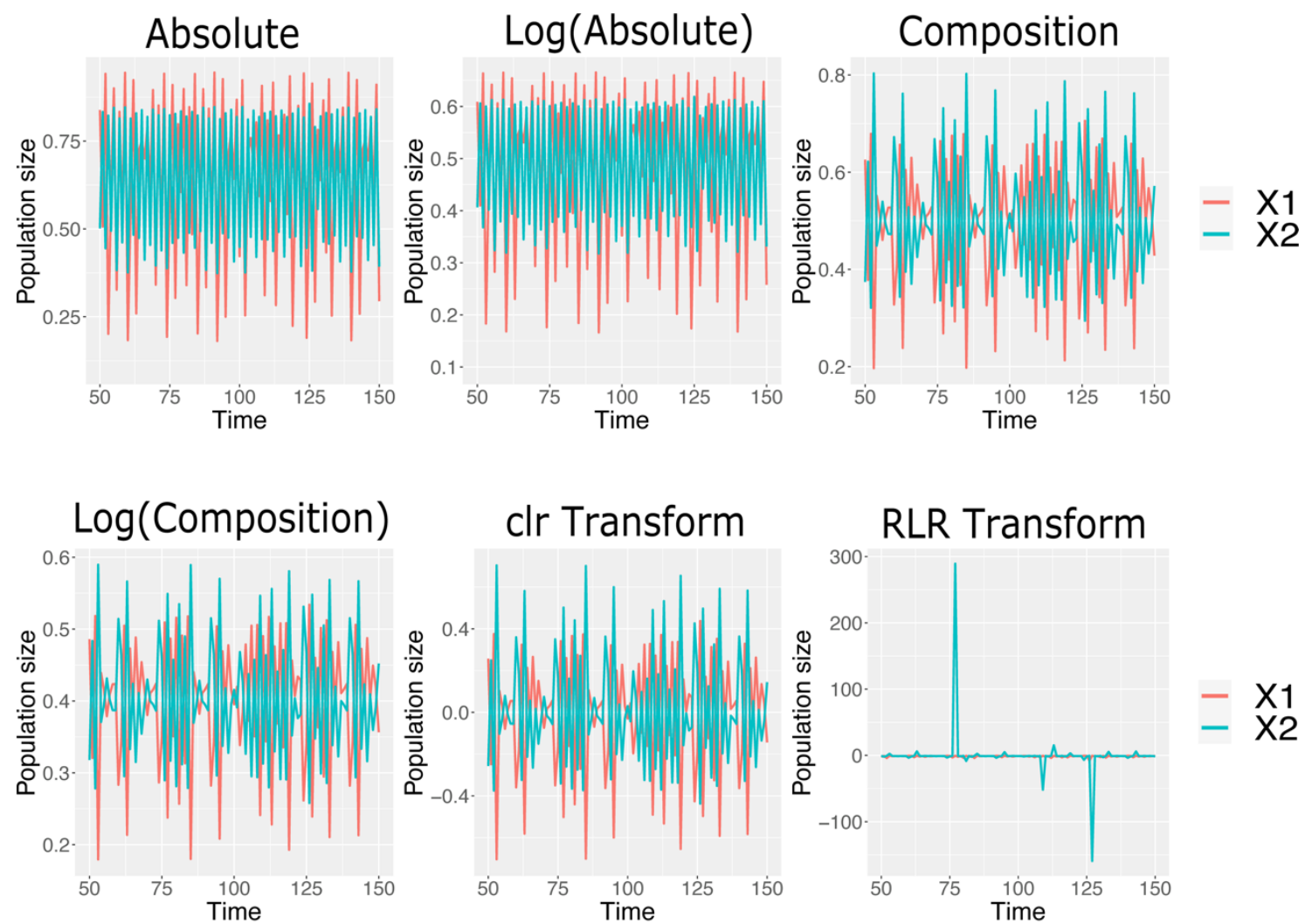

Fig S1. Changes for five transformations with reference to "Absolute."

635 "Composition," "Log Composition," and "clr-Transform" has a constant sum of the two 
636 time-series datasets. By contrast, no constant sum is found for "Absolute," "Log

637 Absolute," and "RLR-Transform."

638

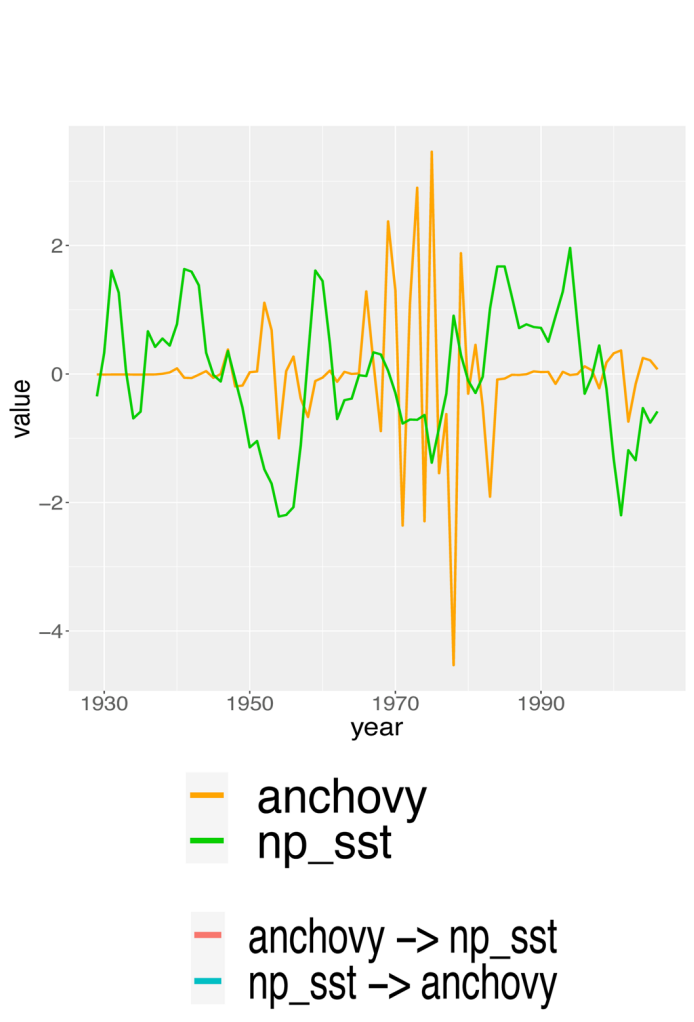

639
(A)

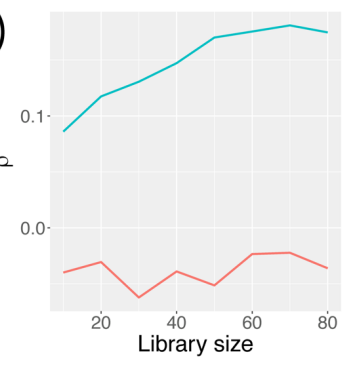

(B)

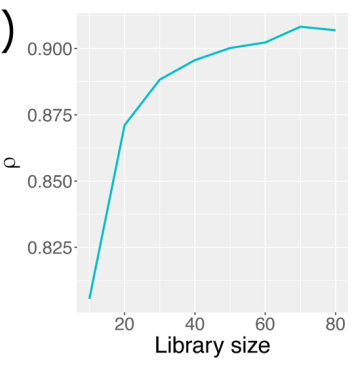

(C)

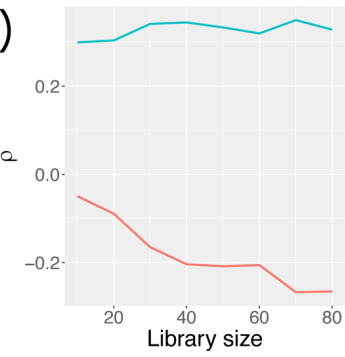

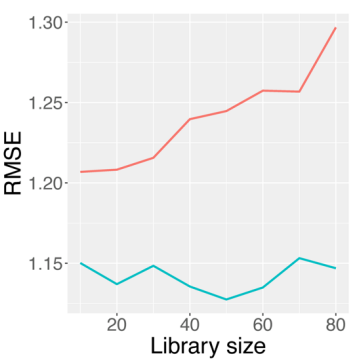
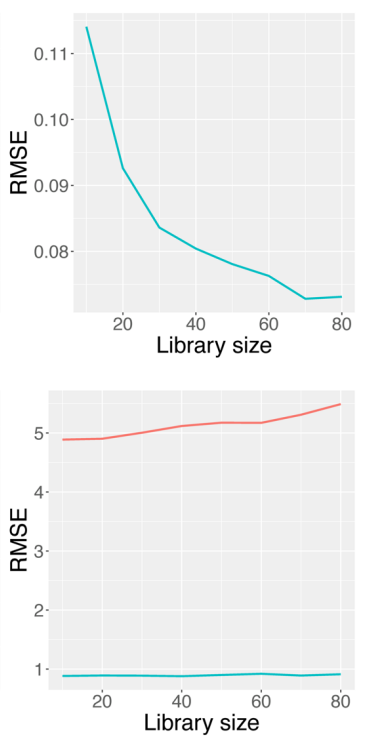

640 Fig S2. CCM results before and after conversion for determining the relationship

641 between northern anchovy (Engraulis mordax) landings and sea surface

642 temperature (SST) measured at Scripps Pier and Newport Pier, California [14].

643 CCM results for min-max normalized raw data (A), composition (B) and RLR-

644 transform (C). Similar results are obtained for (A) and (C)

646 File S1. All the script file used in this study. 
bioRxiv preprint doi: https://doi.org/10.1101/2021.01.25.428037; this version posted January 26, 2021. The copyright holder for this preprint (which was not certified by peer review) is the author/funder, who has granted bioRxiv a license to display the preprint in perpetuity. It is made available under aCC-BY-ND 4.0 International license.

647 Each R script is used to generate the corresponding figure. 